\title{
BMJ Open Effect of religious involvement on cognition from a life-course perspective: protocol for a systematic review and meta-analysis
}

\author{
Shera Hosseini, Ashok Chaurasia, Martin Cooke, Mark Oremus
}

To cite: Hosseini $S$, Chaurasia A, Cooke M, et al. Effect of religious involvement on cognition from a life-course perspective: protocol for a systematic review and metaanalysis. BMJ Open 2016;6: e011301. doi:10.1136/ bmjopen-2016-011301

- Prepublication history and additional material is available. To view please visit the journal (http://dx.doi.org/ 10.1136/bmjopen-2016011301).

Received 27 January 2016 Revised 6 August 2016 Accepted 17 August 2016

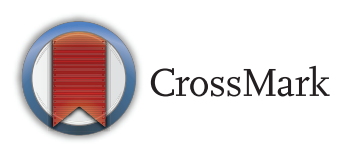

School of Public Health and Health Systems, University of Waterloo, Waterloo, Ontario, Canada

Correspondence to Dr Mark Oremus; moremus@uwaterloo.ca

\section{ABSTRACT}

Introduction: Preserving cognitive health is a crucial aspect of healthy ageing. Both abnormal and normal cognitive decline can adversely affect the health of ageing populations. Evidence suggests religious involvement (RI) can preserve cognition in ageing populations. The primary purpose of this review is to examine the evidence regarding the association between $\mathrm{Rl}$ and cognition from a life-course perspective.

Methods and analysis: This systematic review and meta-analysis has been registered with PROSPERO (registration number CRD42016032331). We will search MEDLINE, PSYCHINFO and EMBASE, and include primary studies with a comparison group, for example, cohort, cross-sectional and case-control studies. To supplement the database search, we will also search the grey literature and the reference lists of included studies. Two reviewers will independently assess and extract data from the articles. Risk of bias and the strength of evidence will be assessed. For sufficiently homogeneous data in domains such as study methods and measures of $\mathrm{RI}$ and cognition, we will pool the results using DerSimonian and Laird meta-analysis.

Ethics and dissemination: Since this is a protocol for a systematic review, ethics approval is not required. The findings of this review will be extensively disseminated through peer-reviewed publications and conference presentations.

\section{BACKGROUND}

The maintenance of cognitive health is an important component of successful ageing. Abnormal cognitive decline is associated with the onset of dementing disorders such as Alzheimer's disease. Even natural cognitive decline in later life can adversely affect health through loss of mobility, increased reliance on assistance to help with performing daily tasks, and reduced quality of life as a result of new limits on one's ability to maintain a certain lifestyle. $^{1}$

\section{Strengths and limitations of this study}

- The major strength of this review will be to study the association between religious/spiritual involvement and cognition from the life-course perspective, which best accounts for the effects of changing social phenomena on health outcomes.

- No agreed on definition of religious/spiritual involvement exists and researchers therefore use many instruments to measure the construct.

- Thus, within this review, we will include a heterogeneous set of exposure measures that could challenge our ability to make comparisons using meta-analysis. The same issue applies to cognition due to the multiplicity of instruments that exist to measure it.

Religious/spiritual involvement (R/SI) may provide a soothing outlet for feelings of stress and depression. Such an outlet can counteract the physiological changes (eg, elevated blood cortisol) associated with experiencing stress and depression. These changes can otherwise negatively affect the areas of the brain that are responsible for memory. ${ }^{2-4}$

Koenig proposes that R/SI can stimulate the higher cortical functions related to abstract thinking and thereby preserve cognitive function. This stimulation occurs because religious practitioners often think about 'higher order' issues such as morality, meaning in life and transcendence. ${ }^{5}$ Hill views R/SI as giving practitioners a greater sense of hope, meaning and purpose in life, all of which can serve as coping mechanisms against stress, anxiety and depression. Reduced strain on one's emotional and mental health can help prevent hippocampal atrophy and cognitive decline. ${ }^{6}$ Hill believes religious practices such as singing, praying, attending sermons, studying scripture and socialising with others during faith-based activities can maintain dense neocortical brain synapses and delay cognitive deterioration in the elderly. 


\section{OBJECTIVES}

We will undertake a systematic review and examine whether R/SI is associated with cognition over the life course in adults (primary research question). The lifecourse perspective envisages a dynamic process whereby intrinsic (eg, biological) and extrinsic (eg, environmental) factors, as well as changes in these factors over time, combine to affect health in later life. ${ }^{7}$ Longitudinal changes in the degree of R/SI and cognition over time, rather than simply assessing $\mathrm{R} / \mathrm{SI}$ at baseline and cognition at some future point, will provide a full appreciation of the association between R/SI and cognition. Changes in other variables over time (ie, functional ability, presence of comorbidities) that can affect the degree of $\mathrm{R} / \mathrm{SI}$ or cognition will also be examined to assess the association of interest.

We will examine two secondary research questions in the review. First, does any form of social engagement serve as a mediator, effect modifier or confounder of the association between R/SI and cognition? Adjusting for social engagement is needed in studies of $\mathrm{R} / \mathrm{SI}$ because R/SI involves a social component (eg, attending services, singing in choirs, serving on committees, attending retreats, taking courses [eg, Bible study]) and researchers should assess whether R/SI has an effect over and above social engagement. ${ }^{8}$

Second, does the association between R/SI and cognition differ (in strength or direction) according to the means of measuring R/SI or cognition? Many measures of R/SI exist, including scales to measure degrees of spirituality or religiosity, ${ }^{10-12}$ attitudes toward Christianity, ${ }^{12}$ degrees of religious self-identity and conviction, ${ }^{8}{ }^{13}$ and religious coping. ${ }^{14} 15$ Additional measures include frequency of viewing religious television and radio programming, ${ }^{13}$ frequency of religious service attendance ${ }^{8} 1316$ and frequency of prayer. ${ }^{13} 17$

Additionally, many different measures of cognition exist. They vary depending on the specific domain of cognition that is intended for assessment, as well as according to the type of test (questionnaire-based scales vs neurocognitive assessments).

\section{METHODS}

This systematic review and meta-analysis has been registered with PROSPERO (registration number CRD42016032331). We developed our methods following the instructions of the Preferred Reporting Items for Systematic Reviews and Meta-Analysis ${ }^{18}$ (PRISMA) guidelines and the systematic review itself will be also written in accordance with the PRISMA and PRISMA-P ${ }^{19}$ statements.

Studies will be selected according to the following eligibility (inclusion/exclusion) criteria.

\section{Study designs}

This review will include primary studies that contain a comparison group (eg, cross-sectional, case-control and cohort). We will exclude narrative or systematic reviews, letters to the editor, abstracts, case series and animal studies.

\section{Participants}

We will include any study of adults (18 years or older) to understand the association between R/SI and cognition from a life-course perspective. Limiting inclusion to studies conducted in the aged (ie, age $\geq 65$ years) would reduce the review's potential to examine important issues such as whether persons who maintain ongoing $\mathrm{R} / \mathrm{SI}$ over time experience better cognitive outcomes than persons who increase or decrease their R/SI at a certain point in life.

\section{Exposure}

We will include any measure of R/SI, including (but not limited to) the measures of R/SI described above.

\section{Outcome}

We will include any means of measuring cognition, including (but not limited to) the measures described below.

Intellectual functioning: Wechsler Adult Intelligence Scale-Revised (WAIS-R), ${ }^{20}$ Stanford-Binet Intelligence Scale-IV ${ }^{21}$

Language processing: Boston Naming Test, ${ }^{22}$ Multilingual Aphasia Examination, ${ }^{23}$ Token Test ${ }^{24}$

Visuospatial processing: Rey-Osterrieth Complex Figure, ${ }^{25}$ Hooper Visual Organisation Test ${ }^{26}$

Attention/concentration: Paced Auditory Serial Addition Test (PASAT), ${ }^{27}$ Serial Sevens ${ }^{28}$

Verbal learning and memory: Wechsler Memory Scale (WMS), ${ }^{29}$ Rey Auditory Verbal Learning Test, ${ }^{30}$ Hopkins Verbal Learning Test ${ }^{31}$

Executive function: Wisconsin Card Sorting Test, ${ }^{32}$ Trail Making Test, ${ }^{33}$ Mini-Mental State Examination (MMSE), ${ }^{34}$ Mental Alternation Test, ${ }^{35}$ Animal Naming Test ${ }^{36}$

Processing speed: Symbol Digit Modalities Test, ${ }^{37}$ Reaction Time Assessments

\section{Timing}

We will include studies published since 1990 onwards and regardless of length of follow-up.

\section{Setting}

Studies undertaken in any setting will be eligible for inclusion (eg, community, hospital, long-term care facility).

\section{Language}

Studies published in any language will be eligible for inclusion. Studies published in any language other than English will be translated into the English language and assessed for eligibility.

\section{Information sources}

We will search MEDLINE (OVID interface, 1990 onwards), EMBASE (OVID interface, 1990 onwards) 
and PSYCHINFO (OVID interface, 1990 onwards). We chose 1990 as the start date because preliminary scoping of the literature suggested no relevant citations would be retrieved prior to 1990 . We will also search the reference lists of included studies. We consulted a medical librarian to develop our search strategy. Our final search terms include textwords (memory [prospective, episodic, declarative], executive function, processing speed, spirituality and church attendance) and exploded subject headings (cogniti*, religio*). The MEDLINE search strategy is included in online supplementary appendix 1. The MEDLINE strategy will be adapted to the syntax and subject headings of the other databases. We will also employ the aforementioned textwords to search the grey literature via Google and will include any studies that report on the association between $\mathrm{R} / \mathrm{SI}$ and cognition.

\section{Study records}

We will use Distiller Systematic Review (DSR) software to facilitate collaboration among our reviewers during the process of study screening. We will use our eligibility criteria to develop screening questions for title/abstract and full-text screening.

\section{Study selection and data extraction}

Two raters will independently screen studies for inclusion based on our eligibility criteria. The raters will perform two levels of screening: (1) title/abstract and (2) full text. Studies that meet the eligibility criteria at the title/abstract screening level, along with studies that the raters cannot confidently call as relevant based on the eligibility criteria, will advance to full-text screening for further consideration. At each screening level, the raters will resolve disagreements by consensus.

Two reviewers will independently extract the following data from each included study: study details (eg, authors, year, country, setting, length of follow-up), sample characteristics (eg, age, sex), study design (eg, casecontrol, cohort, cross-sectional), descriptions of how $\mathrm{R} / \mathrm{SI}$ and cognition are measured, list of covariates included in regression models and study outcomes. The reviewers will contact study authors to address any uncertainties regarding the data they wish to extract.

To ensure consistency in screening and data extraction across multiple personnel, we will develop standardised instructions and programme web forms into DSR. We will pretest the instructions and forms on 20 randomly selected studies.

\section{Assessment of risk of bias}

Two raters will use the Newcastle-Ottawa Scale (NOS) (http://www.ohri.ca/programs/clinical_epidemiology/ oxford.asp) to independently assess the risk of bias of the included studies. The raters will resolve discrepancies through consensus. The NOS assesses three domains, including selection of study groups, comparability of study groups and detection of outcome. The scale comprises two subscales, one geared toward cohort studies and one designed for case-control studies. The NOS has also been adapted for use with cross-sectional studies.

\section{Grading strength of evidence}

To evaluate the strength of evidence on the topic, we will use the Grading of Recommendations Assessment, Development and Evaluation (GRADE) approach. ${ }^{38}$ Two raters will independently assess the strength of evidence in five areas: study design, quality, consistency directness and precision. The raters will add or subtract points for each of these categories in line with GRADE guidelines. By adding or subtracting points, GRADE helps us assess whether further evidence from newly published studies would change the conclusions of the review.

\section{Data synthesis and statistical analysis}

We will narratively synthesise the extracted data ${ }^{39}$ from all included studies. For subsets of studies that are sufficiently homogeneous in terms of sample characteristics, measures of $\mathrm{R} / \mathrm{SI}$ and cognition, and methods (eg, design, setting, length of follow-up), we will pool results using DerSimonian and Laird's random effects meta-analysis model, ${ }^{40}$ implemented through R V.3.2.2 statistical software (R Foundation for Statistical Computing, Vienna, Austria).

\section{DISCUSSION}

The systematic review will provide the most comprehensive assessment of the state of knowledge on the association between R/SI and cognition to date. The major benefit of this review will be to study the association from the life-course perspective, which best accounts for the effects of changing social phenomena on health outcomes. This perspective views ageing as a dynamic process whereby individuals are impacted by changing intrinsic (eg, biological) and extrinsic (eg, environmental) factors over time. These factors may directly affect individuals' health as they age. In addition, these factors could prompt behavioural and lifestyle changes that also affect health over time. From a life-course perspective, longitudinal changes in the association between R/SI and cognition are important, as are changes in other variables (i e, functional ability, presence of comorbidities) that can affect the degree of R/SI or cognition over time. We anticipate that this review will uncover valuable information regarding the potential enhancing effect of $\mathrm{R} / \mathrm{SI}$ on cognition.

Some potential shortcomings of this review should be mentioned. No agreed on definition of R/SI exists and researchers therefore use many instruments to measure the construct. Thus, within this review, we will include a heterogeneous set of exposure measures that could challenge our ability to make comparisons using meta-analysis. The same issue applies to cognition due to the multiplicity of instruments that exist to measure it. 
Agli et $a t^{41}$ recently published a systematic review of studies examining R/SI and dementia. In comparison to Agli $e t a l$ s review, our systematic review will seek to examine the evidence for the link between R/SI and cognition in any population regardless of cognitive or disease status (ie, normal cognition, mild/moderate cognitive decline, severe cognitive decline or dementia). Our proposed systematic review will therefore include a much broader population than Agli et als review.

Acknowledgements The authors wish to thank Jackie Stapleton for helping to develop the literature search strategy.

Contributors SH wrote the protocol and will lead the conduct of the systematic review. MO conceived the study and revised the protocol for important intellectual content. $\mathrm{AC}$ and $\mathrm{MC}$ revised the protocol for important intellectual content. MO, AC and MC will provide conceptual and methods guidance throughout the review. All of the authors have read and approved the final submitted protocol.

Funding This review will be funded by the Network for Ageing Research at the University of Waterloo.

Competing interests None declared.

Provenance and peer review Not commissioned; externally peer reviewed.

Open Access This is an Open Access article distributed in accordance with the Creative Commons Attribution Non Commercial (CC BY-NC 4.0) license, which permits others to distribute, remix, adapt, build upon this work noncommercially, and license their derivative works on different terms, provided the original work is properly cited and the use is non-commercial. See: http:// creativecommons.org/licenses/by-nc/4.0/

\section{REFERENCES}

1. Aging and preventative health. Cleveland Clinic Website. http://www. clevelandclinicmeded.com/medicalpubs/diseasemanagement/ preventive-medicine/aging-preventive-health/Published 2013. (accessed 2 Dec 2015).

2. Conrad CD. Chronic stress-induced hippocampal vulnerability: the glucocorticoid vulnerability hypothesis. Rev Neurosci 2008;19:395-411.

3. Sapolsky RM. Glucocorticoids and hippocampal atrophy in neuropsychiatric disorders. Arch Gen Psychiatry 2000;57:925-35.

4. Csernansky JG, Dong H, Fagan AM, et al. Plasma cortisol and progression of dementia in subjects with Alzheimer-type dementia. Am J Psychiatry 2006;163:2164-9.

5. Koenig HG. Religion, spirituality, and health: the research and clinical implications. Int Sch Res Not 2012;e278730.

6. Hill TD. Religious involvement and healthy cognitive aging: patterns, explanations, and future directions. J Gerontol A Biol Sci Med Sci 2008;63:478-9.

7. Kuh D, Ben-Shlomo Y, Lynch J, et al. Life course epidemiology. J Epidemiol Community Health 2003;57:778-83.

8. Van Ness PH, Kasl SV. Religion and cognitive dysfunction in an elderly cohort. J Gerontol B Psychol Sci Soc Sci 2003;58:S21-9.

9. Hill TD, Burdette AM, Angel JL, et al. Religious attendance and cognitive functioning among older Mexican Americans. J Gerontol B Psychol Sci Soc Sci 2006;61:3-9.

10. Kaufman $\mathrm{Y}$, Anaki $\mathrm{D}$, Binns $\mathrm{M}$, et al. Cognitive decline in Alzheimer disease Impact of spirituality, religiosity, and QOL. Neurology 2007;68:1509-14.

11. Lucchetti G, Lucchetti AGL, Badan-Neto AM, et al. Religiousness affects mental health, pain and quality of life in older people in an outpatient rehabilitation setting. J Rehabil Med 2011;43:316-22.

12. Coin A, Perissinotto E, Najjar M, et al. Does religiosity protect against cognitive and behavioral decline in Alzheimer's dementia? Curr Alzheimer Res 2010;7:445-52.

13. Koenig HG, George LK, Titus P. Religion, spirituality, and health in medically ill hospitalized older patients. J Am Geriatr Soc 2004:52:554-62.
14. Pargament KI, Koenig HG, Tarakeshwar N, et al. Religious coping methods as predictors of psychological, physical and spiritual outcomes among medically ill elderly patients: a two-year longitudinal study. J Health Psychol 2004;9: 713-30.

15. Koenig HG, Cohen HJ, Blazer DG, et al. Religious coping and depression among elderly, hospitalized medically ill men. Am J Psychiatry 1992;149:1693-700.

16. Reyes-Ortiz CA, Berges IM, Raji MA, et al. Church attendance mediates the association between depressive symptoms and cognitive functioning among older Mexican Americans. J Gerontol A Biol Sci Med Sci 2008;63:480-6.

17. Inzelberg R, Afgin AE, Massarwa M, et al. Prayer at midlife is associated with reduced risk of cognitive decline in Arabic women. Curr Alzheimer Res 2013;10:340-6.

18. Liberati A, Altman DG, Tetzlaff $\mathrm{J}$, et al. The PRISMA statement for reporting systematic reviews and meta-analyses of studies that evaluate healthcare interventions: explanation and elaboration. $B M J$ 2009;339:b2700.

19. Shamseer L, Moher D, Clarke M, et al., PRISMA-P Group. Preferred reporting items for systematic review and meta-analysis protocols (PRISMA-P) 2015: elaboration and explanation. BMJ 2015;349: g7647.

20. Wechsler D. Manual for the Wechsler Adult Intelligence ScaleRevised. New York: Psychological Corporation, 1981.

21. Thorndike RL, Hagen EP, Sattler JM. Stanford-Binet Intelligence Scale: fourth edition. Chicago: Riverside, 1986a.

22. Kaplan E, Goodglass H, Weintraub S. Boston naming test. Philadelphia: Lea Febiger, 1983

23. Benton AL, Hamsher K deS. Multilingual aphasia examination. rev. edn. Iowa City: Department of Neurology, University of lowa Hospitals, 1983.

24. De Renzi E, Vignolo LA. The token test: a sensitive test to detect receptive disturbances in aphasics. Brain 1962;85:665-78.

25. Rey A. L'examen psychologique dans les cas d'encephalopathie traumatique. Arch Psychol 1941;28:286-340.

26. Western Psychological Services. Hooper Visual Organization Test (VOT): manual. Los Angeles: Western Psychological Services, 1983.

27. Gronwall D, Sampson H. The psychological effects of concussion. Auckland University Press, 1974.

28. Hayman M. Two minute clinical test for measurement of intellectual impairment in psychiatric disorders. Archives of Neurology and Psychiatry 1942;47:454-64.

29. Wechsler D. Wechsler Memory Scale-third edition manual. San Antonio, TX: Psychological Corporation, 1997.

30. Rey A. L'examenclinique en psychologie [The psychological examination]. Paris: Presses Universitaires de France, 1958.

31. Brandt J. The Hopkins verbal learning test: development of a new memory test with six equivalent forms. Clin Neuropsychol 1991:5:125-42.

32. Heaton RK, Chelune GJ, Talley JL, et al. Wisconsin card sorting test manual. Odessa, Florida: Psychological Assessment Resources, 1993.

33. Reitan RM. Trail making test: manual for administration and scoring. Tucson, AZ: Reitan Neuropsychology Laboratory, 1992.

34. Folstein MF, Folstein SE, McHugh PR. 'Mini-mental state': a practical method for grading the cognitive state of patients for the clinician. J Psychiatr Res 1975;12:189-98.

35. Teng EL. The mental alternation test. Los Angeles, CA: Department of Neurology, University of Southern California School of Medicine, 1994.

36. Rosen WG. Verbal fluency in aging and dementia. J Clin Neuropsychol 1980;2:135-46.

37. Smith A. Symbol digits modalities test: manual. Western Psychological Services, 1982.

38. Guyatt G, Oxman AD, Akl EA, et al. GRADE guidelines: 1. IntroductionGRADE evidence profiles and summary of findings tables. J Clin Epidemiol 2011;64:383-94.

39. Popay J, Roberts $\mathrm{H}$, Sowden A, et al. Guidance on the conduct of narrative synthesis in systematic reviews: a product from the ESRC methods programme. Swindon, UK: Economic and Social Research Council, 2006.

40. DerSimonian R, Laird N. Meta-analysis in clinical trials. Control Clin Trials 1986;7:177-88.

41. Agli O, Bailly N, Ferrand C. Spirituality and religion in older adults with dementia: a systematic review. Int Psychogeriatr 2015:27:715-25. 